A Latent Pharmacokinetic Time Profile to Model Dose-Response Survival Data

Peer-reviewed author version

JACOBS, Tom; STRAETEMANS, Roel; MOLENBERGHS, Geert; Bouwknecht, Adriaan J. \& BIJNENS, Luc (2010) A Latent Pharmacokinetic Time Profile to Model Dose-Response Survival Data. In: JOURNAL OF BIOPHARMACEUTICAL STATISTICS, 20(4). p. 759-767.

DOI: $10.1080 / 10543401003618249$

Handle: http://hdl.handle.net/1942/11000 


\title{
A Latent Pharmacokinetic Time Profile to Model Dose-response Survival Data
}

\author{
Tom Jacobs ${ }^{1}$, Roel Straetemans ${ }^{2}$, Geert Molenberghs ${ }^{1}$, \\ J. Adriaan Bouwknecht ${ }^{2}$, Luc Bijnens ${ }^{2}$ \\ ${ }^{1}$ Interuniversity Institute for Biostatistics and statistical Bioinformatics, Universiteit Hasselt, \\ Agoralaan 1, B3590 Diepenbeek, Belgium, and Katholieke Universiteit Leuven, Belgium \\ ${ }^{2}$ Johnson and Johnson Pharmaceutical Research and Development, \\ a division of Janssen Pharmaceutica N.V., Turnhoutseweg 30, B-2340 Beerse, Belgium \\ running title: A K-PD Model for Dose-response Survival Data
}

SUMMARY

The accelerating rotarod test is a pre-clinical pharmacodynamic test to assess the effect of a treatment on an animal's motor coordination. Two models are proposed to analyze the dose-response time-to-event data that typically result from such experiments: (1) a linear regression model and (2) a $E_{\text {max }}$ model with latent drug concentration at the site of action. Both cope with the survival-character of the data. The latter model allows a direct comparison of compounds, but raises the question whether the study design would benefit from the inclusion of additional mice for plasma concentration sampling on the one hand or whether additional time-to-event data without plasma concentration sampling should be ascertained from these additional mice on the other hand. A simulation study explores the impact on operational characteristics of this change of study design.

Keywords: Accelerated failure time, Accelerating rotarod, Dose-response, K-PD model, Pharmacokinetics.

\section{Introduction}

The development of new medicines requires an enormous amount of research. After potentially promising molecules have been synthesized, they are first tested in vitro, followed by tests in animals and finally humans. Each of these steps should enable to select the 
compounds with a minimum of unacceptable toxicity and a maximum of effect, but without putting animals and patients unnecessary at risk. Therefore, it is important to learn as much as possible with a minimum of in vivo testing.

One of such in vivo tests with mice is the accelerating rotarod test. It assesses the impact of the new treatment on the motor coordination (Gerald and Gupta, 1977, McIlwain et al. 2001). The treatment effect on the mice is measured by evaluating the duration that the mice stay on top of a circular rod that rotates at accelerating speed from 4 to 40 RPM for the first $5 \mathrm{~min}$ and then remains at $40 \mathrm{RPM}$ for another $5 \mathrm{~min}$ (McIlwain et al. 2001). To acquaint the animals to the rod, the study starts with a training phase, followed by a test phase. The animals are placed four times on the rod at 30-minutes intervals during the training phase to allow repeated experience leading to enhanced performance. The second phase corresponds to a dose-response study of the effects on the motor coordination for two compounds over time. These two compounds (phencyclidine (PCP) and d-amphetamine) are expected to disrupt performance on the rod due to their impact on motor coordination. In the experiment considered here, the evaluative occasions are: before administration, at 30, 60, and 90 minutes after administration. Four doses are evaluated for both compounds: $0.0,2.5,5.0$, and $10.0 \mathrm{mg} / \mathrm{kg}$ subcutaneously. A total of 80 mice are included in the study. All animals enter both phases. Ten mice are attributed to each compound-by-dose level combination.

The data were modelled using an accelerated failure time model (Klein and Moeschberger 1997). The shape and scale parameters were fitted using a simple linear model. However, as Holford (2006) states, the causal factor in generating a response is not a dose, but rather the resulting drug concentration at the site of action, such as a receptor. Therefore, also a more elaborate $E_{\max }$ model with a latent pharmacokinetic profile is fitted. This is sometimes referred to as a K-PD model (Jacqmin et al. 2007). This model raises the obvious question as to whether sampling of plasma concentrations would yield more accurate dose-response estimates. A simulation study is set up to assess the impact of an increase of the sample size on the accuracy of the estimation. It is hoped that the use of a few additional animals for pharmacokinetic sampling would enable a better estimation of the dose- and time-related 
toxic effects and as such would lower the risks when making the transition to humans.

The remainder of the paper is organized as follows. The dose-response models are presented in Section 2. The case study is discussed in Section 3. The set up of the simulation study is described in Section 4.

\section{Methodology}

Gerald and Gupta (1977) analyzed their data using medians and Wilcoxon tests. They do not mention how censored data were handled. In this section, we propose a more formal statistical framework to take into account both the censoring and the dose response.

The response of interest is of the time-to-event type with event falling off the rod. The survival function, $S(t)$, characterizes the distribution of this response and is the probability of having a time-to-event beyond $t$ (Klein and Moeschberger 1997). The time to event data are analyzed using an accelerated failure time (AFT) model where the natural logarithm of the time to event is modelled as a function of covariates. The first step in developing the AFT model is the choice of the distribution. For the given experiment, the Weibull distribution was chosen, based on the training dataset.

$$
S(t)=\exp \left[-(\alpha t)^{\gamma}\right]
$$

and where $\alpha>0$ is the scale parameter, $\gamma>0$ the shape parameter, and $t$ the time to falling off the rod. Instead of modelling $\alpha$ and $\gamma$, the following transformation is applied: $\alpha=\exp \left(-\alpha^{\prime}\right)$ and $\gamma=\exp \left(-\gamma^{\prime}\right)$ to ensure $\alpha>0$ and $\gamma>0$. Further, we define $T_{i}$ as the evaluation time $i$ during the testing phase; $D_{j}$ correspond to the dose level $j$. The right-censoring of the data is included in the likelihood function $L$ as described in Klein and Moeschberger (1997).

To calculate the cumulative distribution function from the density $g(t)$, one needs to integrate over time. The parameters $\alpha$ and $\gamma$ are a function of time and should be included in the integration. However, the repeated time-to-event evaluations take only a limited amount of time in comparison to the entire experiment, or more precisely to the 
underlying pharmacokinetic profile. Therefore, one could consider that the parameters $\alpha$ and $\gamma$ are approximately constant during each time-to-event assessment. The integration and likelihood simplify to (2.2).

$$
\begin{aligned}
G(t) & =\exp \left[-(\alpha t)^{\gamma}\right] \\
g(t) & =\gamma \alpha\left[(\alpha t)^{\gamma-1}\right] G(t), \\
\log [L(t)] & =1_{o b s} \log [g(t)]+1_{\text {cens }} \log [G(t)],
\end{aligned}
$$

where cens denotes a right-censored observation, and obs indicates that an event was observed. The log-likelihood function was implemented in the procedure NLMIXED using the SAS software.

The dose-response relation is incorporated using two different models: the first model is the linear regression model, where dose is included as a linear effect and time as a quadratic effect. The interaction effect is included as well. Time is expected to be quadratic, because no effect is expected at the time of administration (time 0), whereas a large effect is expected shortly after administration (time 30), fading out at later time points, hence the quadratic term. The interaction is needed because one cannot automatically expect the same dose effect at each point in time, nor can the same time effect be expected for all doses. Table 1 contains the different parameters, which allows for a better understanding of the model.

A more conventional way of modelling a dose-response relationship in pharmacology is the so-called $E_{\max }$ model (Gabrielson and Weiner 2000). It assumes that the change in time to falling off the rod depends on the underlying drug concentrations of the compounds. These latent drug concentrations at the site of action are included in the model in a sigmoidal way, i.e., an $E_{\max }$ model is fitted for both the scale and shape parameters assuming a latent one-compartmental model with first-order absorption (Jacqmin et al. 2007):

$$
\begin{aligned}
\alpha_{i j k}^{\prime} & =\beta+\frac{e_{\max } c_{i j}}{\exp \left(e c_{50}\right)+c_{i j}}, \\
\gamma_{i j k}^{\prime} & =\beta_{\gamma}+\frac{e_{\max _{\gamma}} c_{i j}}{\exp \left(e c_{50_{\gamma}}\right)+c_{i j}},
\end{aligned}
$$

where $c_{i j}$ stands for

$$
c=\frac{D_{j} \exp \left(\phi_{2}\right)}{\exp (V)\left[\exp \left(\phi_{2}\right)-\exp \left(\phi_{1}\right)\right]}\left\{\exp \left[-\exp \left(\phi_{1}\right) T_{i}\right]-\exp \left[-\exp \left(\phi_{2}\right) T_{i}\right]\right\} .
$$


Here, $e_{\max }$ is the maximal asymptotic effect, and $e c_{50}$ the concentration at which $50 \%$ of the maximal effect is attained. $\alpha_{i j k}^{\prime}$ and $\gamma_{i j k}^{\prime}$ correspond to the scale and shape parameter of the Weibull distribution, respectively, where different values for $e_{\max }$ and $e c_{50}$ are used in the scale and the shape parameter. $\phi_{1}$ and $\phi_{2}$ are the rate constants determining the latent drug concentration at the site of action model. $D_{j}$ and $T_{i}$ still indicate the dose level $j$ and the time point $i . \quad c_{i j}$ stands for the latent drug concentration. This latent drug concentration is considered to correspond to the concentration at the place of action, e.g., the receptors in the brain or in the bloodstream. The parametrization used ensures that positive values are obtained for $\exp \left(e c_{50}\right), \exp \left(\phi_{1}\right), \exp \left(\phi_{2}\right)$, and $\exp (l v f)$. It also acknowledges the lognormal distribution of the quantities. The number of parameters to be estimated is reduced compared to previous models. Additionally, the model has a pharmacological basis. The dependence of the data, i.e., each mouse is tested four times in the test phase, can be taken into account by the inclusion of a random effect at $e c_{50}, e c_{50_{\gamma}}, \phi_{1}$, and/or $\phi_{2}$. Unfortunately, the small sample size prohibited the inclusion of a random effect in the test phase of our case study.

The median time to event can be calculated using the following expression, applicable to the case of a Weibull distribution (Klein and Moeschberger 1997):

$$
\frac{\Gamma(1+1 / \gamma)}{\alpha^{1 / \gamma}}
$$

\section{Analysis of the Case Study}

The case study consists of two parts: the training phase to acquaint the animals to the rod, and the test phase. No treatment is administered during the training phase, but all 80 mice are placed four times on the rod. The mice are subsequently split into two groups and one compound is tested per group of 40 animals. Ten mice are then attributed per dose level $(0$, 2.5, 5, and $10 \mathrm{mg}$ ) and tested before, and 30,60, and 90 minutes after administration. It is favorable to estimate system parameters such as $E_{\max }$ in the K-PD model simultaneously over different compounds to improve the estimation. However, this assumes that the compounds share the same method of action, such as blocking the same receptor. In our case study, the 
compounds have different methods of action, which leads to different maximal attainable effects. Therefore, the data of the training phase were analyzed as a whole, the test phase was analyzed per compound.

A Weibull distribution fits the training data best in contrast to the log-logistic and log-normal distribution (Akaike Information Criterion (AIC): 3265.7, 3379.0, and 3277.3, respectively). A random intercept is included for the training data to cope with the dependency of the measurements between occasions. The Weibull distribution seems most appropriate and this distribution will also be used for the test phase. In the test phase, the sample size was too small to fit a random effect in the models described in the rest of the paper.

The test data are analyzed using a linear regression model and a pharmacological model for both compounds separately. The first model has both the scale and shape parameter fitted with linear dose and quadratic time incorporated. The interaction terms are included apart from dose-by-time ${ }^{2}$ in the shape parameter $\gamma^{\prime}$. Addition of the latter resulted in failure of the model's convergence. For the second model, the scale and shape parameter are fitted using an $E_{\max }$ model. An underlying, latent, one compartmental latent drug concentration-time profile at the site of action is estimated (Jacqmin et al. 2007).

AIC values indicate that the second model gave a superior fit for both compounds: 1445.8 and 1427.8 for compound A, and 1489.8 and 1475.5 for compound B for the linear and $E_{\text {max }}$ models, respectively. The model fit of both models is presented in Figures 1 for compound A. It illustrates superiority of the $E_{\max }$ model. The dashed line in Figure 1 shows the inadequate fit of the linear regression model for the higher doses at 30 seconds, whereas the $E_{\max }$ model performs well.

The parameter estimates for the two models can be found in Table 1. The parameters of the linear model do not allow a direct physiological interpretation. One can generally say that a reduction of the scale parameter $\alpha^{\prime}$ is translated into a shorter latency to fall off the rod. However, also the change of the shape parameter $\gamma^{\prime}$ has an impact on the median time-to-event. Although the coefficients of $T$ and $T^{2}$ are small, it has an important impact 
on the fit, as time $T$ is expressed in minutes. The quadratic term of $T$ allows for a high time-to-event, both at the start (before administration) and the end of the study at 90 minutes, whereas a short time-to-event is obtained after 30 minutes. The interaction of time with the dose is needed to enforce that there is no change over time for the placebo dose, simultaneously allowing for a significant reduction at 30 minutes. The parameter estimates of the $E_{\max }$ model are easier to interpret, as mentioned in Section 2. Both the potency $\left(E C_{50}\right)$ and the maximal effect emax are directly obtained.

Figure 2 contains the model-predicted median reduction of the time to falling for both models for compound A. The dose-response relationship is readily visible in the figure, with a peak effect at 30 minutes.

\section{Simulation Study}

The observation that a latent pharmacokinetic time profile can be estimated and that it improves the fit compared to a more traditional linear model is worth further scrutiny. Therefore, a simulation study is set up to explore the added value of increasing the number of mice in the study before discussing the results of the experiment. Such additional mice can theoretically be used for plasma concentration sampling or for additional time-to-event data. In the latter case, the additional mice can be attributed to the existing dose levels, or, alternatively, to higher dose levels for a better exploration of the dose-response curve.

A Weibull distribution is used to simulate four doses $(0,2.5,5$, and 10) at four time points (before administration, 30, 60, and 90 minutes after administration). Both the shape and scale parameter of the Weibull distribution exhibit a sigmoidal behavior with a latent drug concentration at the site of action. Five hundred studies are simulated per situation. The gain of sampling a number of plasma concentrations in additional mice is assessed by plotting the accuracy of the estimates as a function of the number of additional plasma samples. The simulation is performed under the assumption that only one blood sample can be taken per mouse. Further, it is assumed that blood sampling would influence the time to falling off the rod. Therefore, an additional mouse is required for each plasma 
concentration sample. The number of plasma concentration samples, and therefore the number of additional mice, is set to vary from 0 up to 12 by steps of 4 due to the number of doses included. The latter would lead to 10 to 13 mice per dose level. The gain of additional mice in the study can as such be explored at three levels: (1) what is the impact on the $E_{\text {max }}$ modelling if these additional mice are used for plasma concentrations sampling on the one hand; (2) what is the impact on the modelling if these mice were not used for plasma concentration sampling but for additional rotarod assessments per dose; and (3) what is the consequence of an additional, higher dose level $(20 \mathrm{mg}) \mathrm{s}$ on the accuracy and precision of the estimates?

Data were simulated according to the proposed latent drug concentration model with and without a measured plasma concentration-time profile. Additionally, a better dose exploration was simulated in a third part of the simulation. Random variation was included in the simulation at two levels; the time-to-event data, and at the latent drug rate or the plasma concentration-time profile. In the simulation, it is assumed that both the latent drug concentration-time profile and the plasma concentration-time profile have a mouse-dependent log-normally distributed elimination. The residual error of the plasma concentration-time profile was assumed to follow a log-normal distribution; there was assumed to be measurement error. Contrary to the plasma concentration-time profile, no measurement error was attributed to the latent drug rate time profile given the latent, i.e., unmeasured, character of the profile.

The assumption of a direct response in this simulation is crucial, i.e., the time of the peak of the plasma concentration-time profile coincides with the time the maximal pharmacodynamic effect. This assumption is required for a fair comparison of the three settings. If the pharmacokinetic and pharmacodynamic peak effect do not coincide, a delay ought to be built in. This is typically achieved with an effects compartment.

Figure 3 contains the boxplot with the parameter estimates from the simulation study. Five hundred studies per situation were simulated. This notwithstanding, model convergence was not attained in a number of studies due to a negative Hessian matrix: only 187, 206, 
225, and 226 of the 500 studies had a positive Hessian if 10, 11, 12, and 13 mice per dose group were used. This means that this model estimation fails in more than half of the times in the current design with the number of mice, doses and time points of sampling. If, on the contrary 1, 2, or 3 mice per dose group were additionally recruited for plasma concentration sampling, leading to a total sample size of 11, 12, and 13 mice per dose group, the number of studies with a positive Hessian matrix increased to 354,450 , and 412, respectively. In case of an additional dose level, the number of studies with a positive Hessian matrix was 236, 264, 242, and 239, respectively. The latent drug concentration-time profile turns out to be difficult to estimate, which should not come as a surprise as it is difficult to estimate an unobserved underlying profile.

It is striking that none of the box plots for each of the parameters shows any large differences. This suggests that a small amount of additional mice for either plasma concentration sampling or time to event data does not have a large impact on the accuracy of the parameter estimation unless the dose range is explored further. The accuracy and the precision is tabulated as the mean and standard deviation of the parameter estimation in Table 2, providing additional detail over the box plots. No major effects are observed for $\beta$ and $\beta_{\gamma}$.

The estimates for the simulations with a latent dose concentration and a dose exploration both have precise estimates, but the estimation is slihtly biased for $E C_{50}, E C_{50_{\gamma}}$, and $V$. This is possibly due to confounding. The plasma sampling simulation has slightly bias estimates for Emax andEmax $\operatorname{Ex}_{\gamma}$. The strong decrease of the standard deviation in the case of extra dose exploration suggests that the inflated variability observed in the case study and both other simulations is due to a limited dose range in study. Therefore, the fact that the parameter Emax is poorly estimated owes to the poor exploration of the dose-response relationship, rather than the modelling technique. A better dose exploration would solve this issue. 


\section{Discussion}

The rotarod test is used to assess the effect in mice on motor coordination of two compounds. The time to fall is measured. Two different models are proposed to model the dose response time-to-event data. Both use a Weibull distribution and are of an accelerated failure type, but the shape and scale parameters include either a linear model or a $E_{\text {max }}$ model with latent drug concentration-time profile at the site of action. The AIC and graphical model fit suggests that the $E_{\max }$ model fits the data better.

Unlike the linear model, the parameter estimates of the $E_{\max }$ model have a physiological interpretation. Both the potency $\left(E C_{50}\right)$ and the maximal effect Emax are directly obtained, which facilitates the comparison of different compounds. The $E_{\text {max }}$ model being the superior model to the data raises the question whether an alternative design would improve the estimation properties. Therefore, a simulation study was set up to investigate the influence on accuracy and precision if 1, 2, or 3 mice per dose group would be added for either time-to-event data, or plasma concentrations. Does the gain in accuracy and precision justify the 4, 8, or 12 additional mice per compound? Alternatively, the impact of a better dose-response exploration on the estimation properties is considered as well. It turns out that the latent drug concentration-time profile is difficult to estimate in the current design, given the number of non-positive Hessian matrices, which is not surprising; only three time points post administration contain too little information to estimate an underlying, unobserved time profile. The accuracy and precision of the parameters $\beta$ and $\beta_{\gamma}$ is not altered much, whereas for the other parameters, the standard deviation is inflated if the additional mice are used for time-to-event data compared to when these mice are used for plasma concentration sampling. In this case, the lack of accuracy is also more pronounced. This confirms the results of Jacqmin et al (2007). However, the inclusion of the higher dose level improves the precision of the estimation of the parameters tremendously. A small bias is however observed in both latent drug concentration simulations Therefore, a better dose-response exploration would be the best option to improve the current design of the study.

A one-compartmental latent drug concentration-time profile with first-order absorp- 
tion is used as a latent time profile of the drug at the site of action. What is the impact of miss-specifying the plasma concentration time profile? In the given design, this is unlikely to have a major impact at the time points the data was collected, because only three assessments are measured after dose administration. Hence, it is unlikely to see, for example, the difference between a one- and a two-compartment model. On the other hand, it would be too demanding for the model to fit such a latent two-compartment drug concentration-time profile given the ten mice per dose group and the three post-administration observations. The impact is considered negligible, because the drug concentration-time profile is only a tool to fit the data with some baseline, followed by a rapid change (increase or decrease) and gradual return to baseline over time.

In conclusion, the study would have benefitted from the addition of a higher dose level to improve the estimation of the dose-response. The addition of minimally four mice for plasma concentration sampling in the $E_{\max }$ model would only be an option if the inclusion of a higher dose level is deemed inappropriate. In that case, historic data might be considered if the data permits. Both would lead to more accurate and precise estimations and as such reduce the risks of bringing the compound into clinical early development. The $E_{\text {max }}$ model with latent drug concentration-time profile at the site of action allows for a direct estimation of the potency of the drug candidate. It would lead to an improved comparison of the compounds and bring the study in the more familiar setting of preclinical pharmacology.

\section{Acknowledgement}

Financial support from the IAP research network nr P6/03 of the Belgian government (Belgian Science Policy) as well as the valuable comments from the reviewers are gratefully acknowledged. 


\section{References}

Gabrielson, J., Weiner, D. (2000) Pharmacokinetic and Pharmacodynamic Data Analysis: Concepts and Applications, Apotekarsocieteten, Stockholm, Sweden.

Gerald, M.C., Gupta, T.K. (1977). The effects of amphetamine isomers on rotarod performance. Psychopharmacology, 55, 83-86.

Holford, N. (2006). Dose response: Pharmacokinetic-pharmacokdynamic approach in Ting, N. . Dose Finding in Drug Development, New York: Springer-Verlag.

Jacqmin, P., Snoeck, E., van Schaick, E., Gieschke, R., Pillai, P., Steimer, J.-L., and Girard, P. (2007). Modelling Response Time Profiles in the Absence of Drug Concentrations: Definition and Performance Evaluation of the KPD Model. Journal of Pharmacokinetics and Pharmacodynamics, 34, 57-85.

Klein, J., Moeschberger, M. (1997). Survival Analysis - Techniques for Censored and Truncated Data, New York: Springer-Verlag.

McIlwain, K.L., Merriweather, M.Y., Yuva-Paylor, L.A., and Paylor, R. (2001). The use of behavioral test batteries: Effects of training history. Physiology and Behavior, 73, 705-717. 
Table 1: Parameter estimates (standard error) for the different models and compounds. The index $\gamma$ indicates that the parameter is part of the shape parameter.

\begin{tabular}{|c|c|c|c|}
\hline Model & Parameter & compound A & compound B \\
\hline \multirow[t]{11}{*}{ linear } & $\beta$ & $7.9784(1.6784)$ & $8.7640(1.6043)$ \\
\hline & $D$ & $-0.3011(0.1595)$ & $-0.2450(0.1719)$ \\
\hline & $T$ & $-0.06011(0.1207)$ & $-0.1190(0.09940)$ \\
\hline & $T^{2}$ & $0.001342(0.001545)$ & $0.001502(0.001201)$ \\
\hline & $D T$ & $-0.00728(0.008242)$ & $0.001677(0.006064)$ \\
\hline & $D T^{2}$ & $-7.12 \mathrm{E}-6(0.000105)$ & $-0.00006(0.000065)$ \\
\hline & $\beta_{\gamma}$ & $-0.2791(0.2053)$ & $-0.3784(0.1827)$ \\
\hline & $D_{\gamma}$ & $0.04925(0.02282)$ & $0.03400(0.02180)$ \\
\hline & $T_{\gamma}$ & $0.004662(0.01296)$ & $0.01557(0.01219)$ \\
\hline & $T_{\gamma}^{2}$ & $-0.00012(0.000153)$ & $-0.00019(0.000145)$ \\
\hline & $D T_{\gamma}$ & $0.000561(0.000346)$ & $0.000364(0.000358)$ \\
\hline \multirow[t]{9}{*}{ Emax } & $\beta$ & $10.2922(1.1088)$ & $10.5209(1.2918)$ \\
\hline & emax & $-16.7595(4.3503)$ & $-9.4220(2.2230)$ \\
\hline & $\log (e c 50)$ & $-4.2316(11.2725)$ & $0.6224(1.8262)$ \\
\hline & $\beta_{\gamma}$ & $-0.5252(0.1070)$ & $-0.5279(0.1230)$ \\
\hline & $e \max _{\gamma}$ & $1.4692(0.6529)$ & $1.7611(0.7990)$ \\
\hline & $\log \left(e c 50_{\gamma}\right)$ & $-4.5599(11.2752)$ & $1.4254(1.9358)$ \\
\hline & $\phi_{1}$ & $-0.7426(32.986)$ & $-2.0620(4.9244)$ \\
\hline & $\phi_{2}$ & $-4.4594(0.3017)$ & $-4.4693(1.3075)$ \\
\hline & $l V f$ & $-2.2085(22.541)$ & $-2.5522(3.7463)$ \\
\hline
\end{tabular}


Table 2: The mean and standard deviation of the parameter estimation in the case of additional mice for time-to-event data, plasma concentration sampling, or an additional dose level.

\begin{tabular}{|c|c|c|c|c|c|c|c|}
\hline \multirow{2}{*}{$\begin{array}{l}\text { parameter } \\
\text { (target value) }\end{array}$} & \multirow{2}{*}{$\begin{array}{c}\text { mice } \\
\text { per dose }\end{array}$} & \multicolumn{2}{|c|}{ time-to-event } & \multicolumn{2}{|c|}{ plasma concentration } & \multicolumn{2}{|c|}{ Dose level } \\
\hline & & mean & STD & mean & STD & mean & STD \\
\hline$\beta$ & 10 & 10.39 & 1.11 & 10.39 & 1.11 & 10.33 & 1.00 \\
\hline \multirow[t]{3}{*}{$(10.29)$} & 11 & 10.56 & 1.13 & 10.52 & 1.16 & 10.48 & 1.02 \\
\hline & 12 & 10.46 & 1.05 & 10.44 & 1.10 & 10.27 & 0.88 \\
\hline & 13 & 10.49 & 1.00 & 10.53 & 1.14 & 10.40 & 0.86 \\
\hline emax & 10 & -21.95 & 20.52 & -21.95 & 20.52 & -17.82 & 2.21 \\
\hline \multirow[t]{3}{*}{$(-16.76)$} & 11 & -19.4 & 6.66 & -20.58 & 16.56 & -17.46 & 1.52 \\
\hline & 12 & -20.51 & 22.03 & -19.84 & 11.27 & -17.44 & 1.59 \\
\hline & 13 & -20.01 & 8.96 & -19.27 & 8.08 & -17.35 & 1.56 \\
\hline $\log (e c 50)$ & 10 & -3.16 & 1.00 & -3.16 & 1.00 & -3.12 & 0.22 \\
\hline \multirow[t]{3}{*}{$(-4.23)$} & 11 & -3.11 & 0.33 & -4.12 & 0.66 & -3.16 & 0.18 \\
\hline & 12 & -3.14 & 0.29 & -4.09 & 0.58 & -3.13 & 0.17 \\
\hline & 13 & -3.13 & 0.32 & -4.13 & 0.58 & -3.16 & 0.16 \\
\hline$\beta_{\gamma}$ & 10 & -0.53 & 0.11 & -0.53 & 0.11 & -0.52 & 0.09 \\
\hline \multirow[t]{3}{*}{$(-0.53)$} & 11 & -0.55 & 0.11 & -0.54 & 0.11 & -0.54 & 0.10 \\
\hline & 12 & -0.54 & 0.10 & -0.53 & 0.10 & -0.52 & 0.08 \\
\hline & 13 & -0.54 & 0.09 & -0.54 & 0.11 & -0.53 & 0.08 \\
\hline \multirow{4}{*}{$\begin{array}{l}\operatorname{emax}_{\gamma} \\
(1.47)\end{array}$} & 10 & 3.08 & 12.88 & 3.08 & 12.88 & 1.41 & 0.27 \\
\hline & 11 & 1.82 & 2.01 & 1.63 & 1.75 & 1.45 & 0.24 \\
\hline & 12 & 1.77 & 2.89 & 1.65 & 2.40 & 1.42 & 0.24 \\
\hline & 13 & 1.82 & 1.53 & 1.61 & 1.06 & 1.46 & 0.26 \\
\hline $\log \left(e c 50_{\gamma}\right)$ & 10 & -3.42 & 1.58 & -3.42 & 1.58 & -3.57 & 0.20 \\
\hline \multirow[t]{3}{*}{$(-4.56)$} & 11 & -3.52 & 0.44 & -4.61 & 0.66 & -3.55 & 0.17 \\
\hline & 12 & -3.59 & 0.48 & -4.58 & 0.61 & -3.56 & 0.17 \\
\hline & 13 & -3.54 & 0.37 & -4.61 & 0.69 & -3.55 & 0.17 \\
\hline$V$ & 10 & 1.39 & 0.73 & & & 1.09 & 0.09 \\
\hline \multirow[t]{3}{*}{$(2.21)$} & 11 & 1.31 & 0.39 & & & 1.08 & 0.09 \\
\hline & 12 & 1.30 & 0.47 & & & 1.09 & 0.09 \\
\hline & 13 & 1.32 & 0.26 & & & 1.08 & 0.08 \\
\hline$\phi_{1}$ & 10 & -0.83 & 1.00 & & & -0.65 & 0.11 \\
\hline \multirow[t]{3}{*}{$(-0.74)$} & 11 & -0.88 & 0.44 & & & -0.66 & 0.09 \\
\hline & 12 & -0.81 & 0.76 & & & -0.65 & 0.10 \\
\hline & 13 & -0.82 & 0.34 & & & -0.66 & 0.09 \\
\hline$\phi_{2}$ & 10 & -4.44 & 0.29 & & & -4.47 & 0.13 \\
\hline \multirow[t]{3}{*}{$(-4.46)$} & 11 & -4.47 & 0.244 & & & -4.46 & 0.14 \\
\hline & 12 & -4.47 & 0.23 & & & -4.47 & 0.11 \\
\hline & 13 & -4.48 & 0.22 & & & -4.48 & 0.12 \\
\hline
\end{tabular}



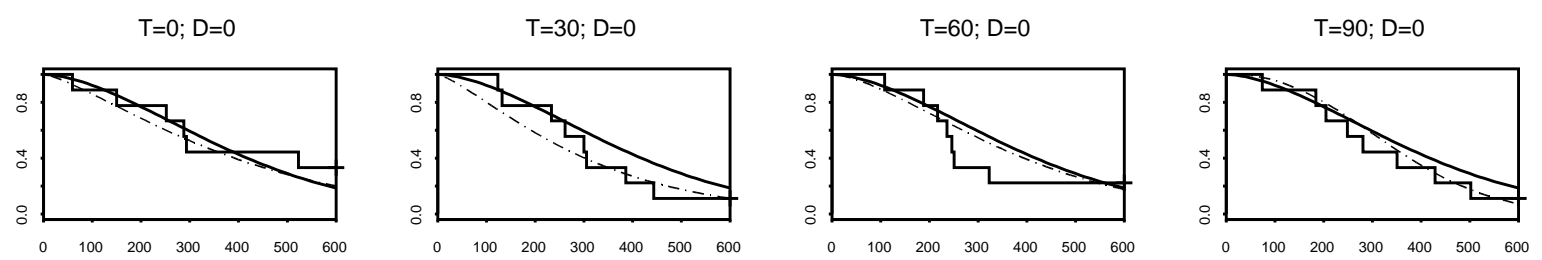

$\mathrm{T}=0 ; \mathrm{D}=2.5$
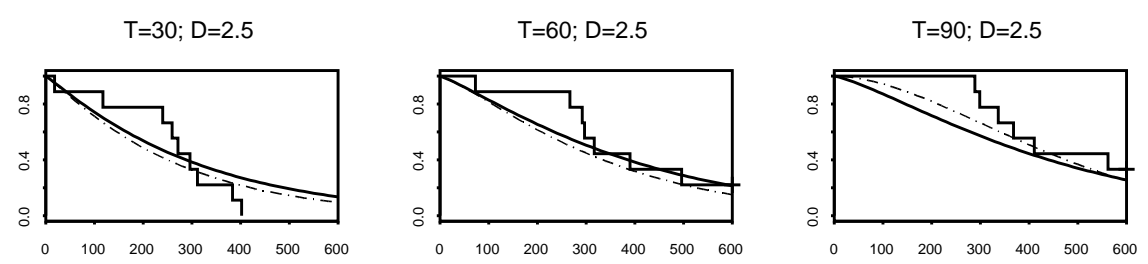

$T=0 ; \mathrm{D}=5$

$\mathrm{T}=30 ; \mathrm{D}=5$

$\mathrm{T}=60 ; \mathrm{D}=5$

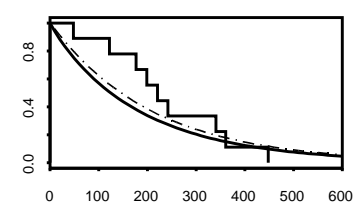

$\mathrm{T}=90 ; \mathrm{D}=5$
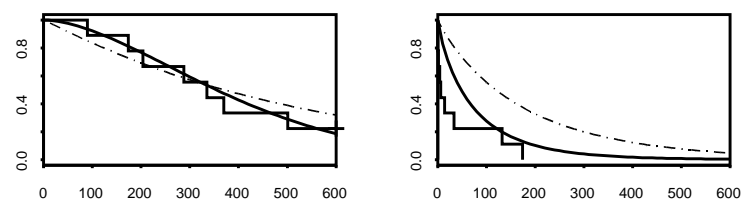

$\mathrm{T}=30 ; \mathrm{D}=10$

$\mathrm{T}=60 ; \mathrm{D}=10$
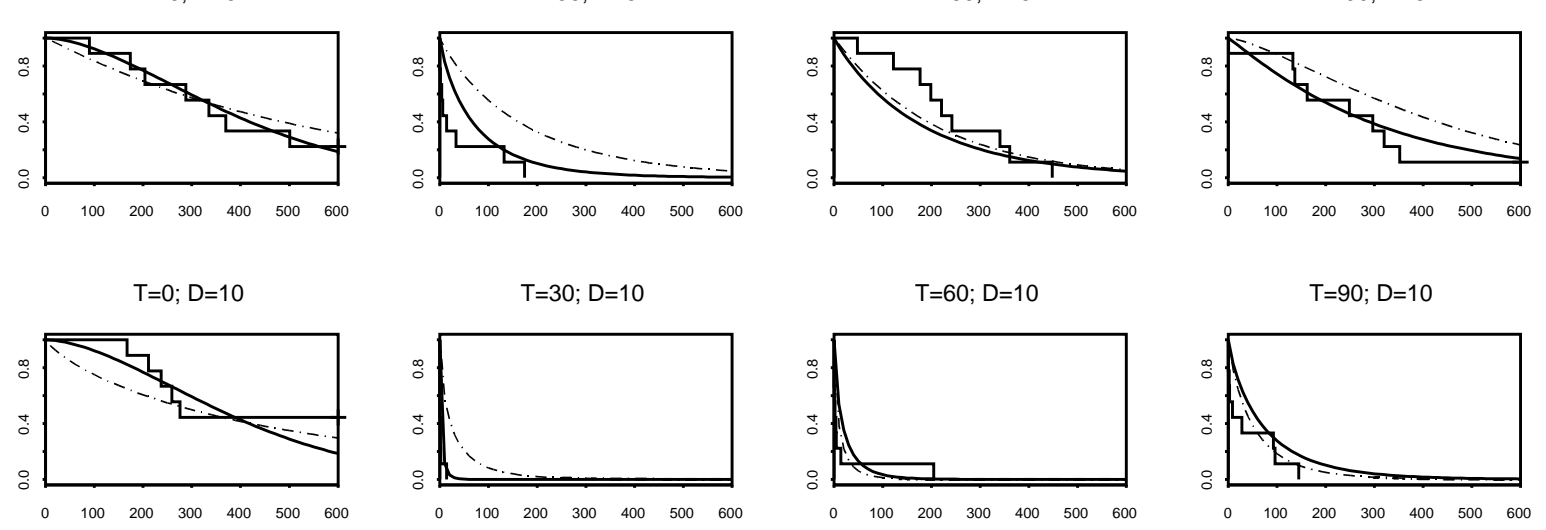

Figure 1: Model prediction of the probability to stay on the rod as function over time (seconds) for compound A for the different doses $D(\mathrm{mg} / \mathrm{kg}$ ) and timepoints (minutes) after administration $T$, where the full line is the Emax model, and dashed is used for the linear model. 


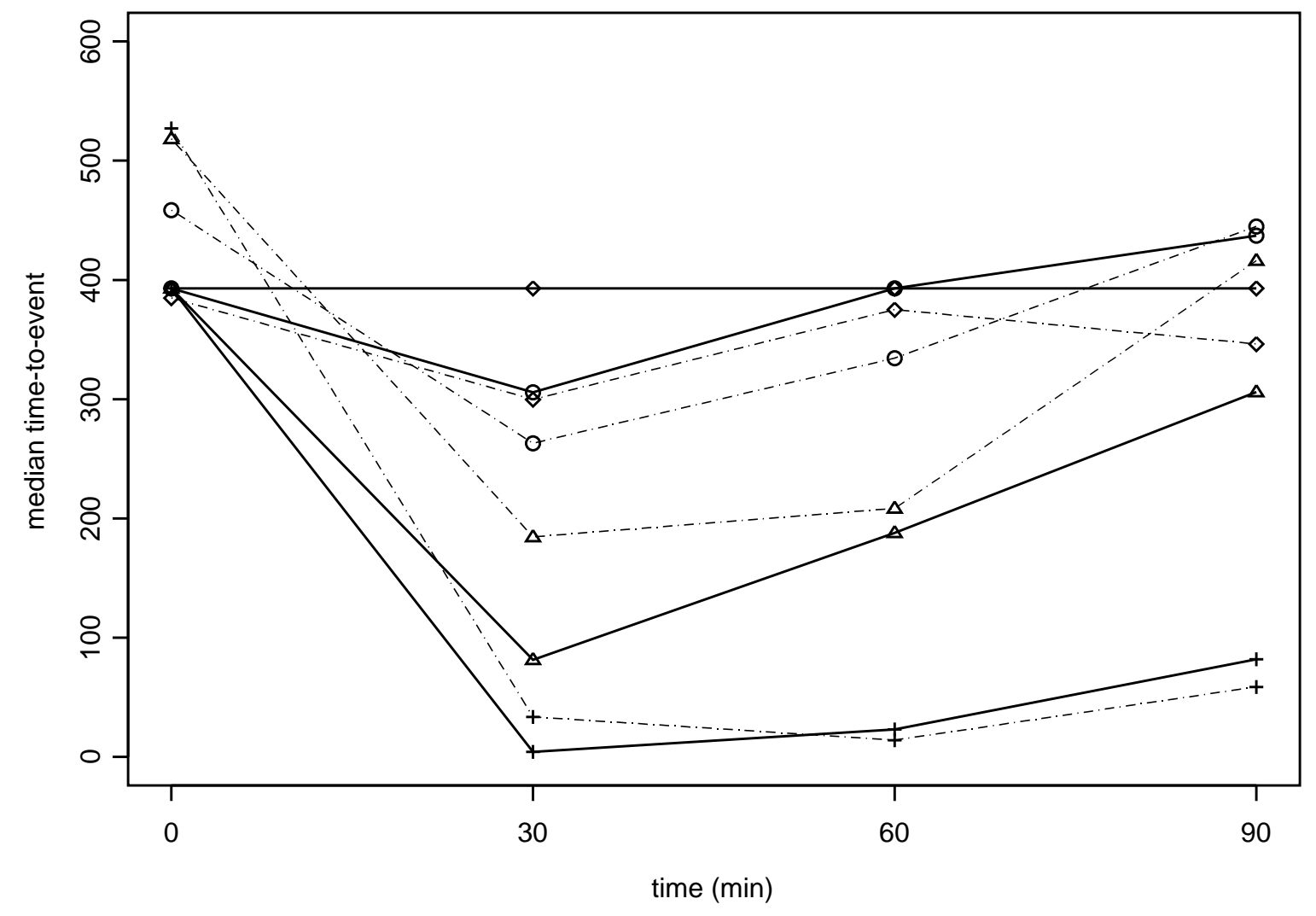

Figure 2: Model based median reduction of time to falling off the rod for compound A, where the full line is the Emax model, and dashed is used for the linear model. Following symbols are used: $\diamond$ for $0 \mathrm{mg} / \mathrm{kg}$, ○ for $2.5 \mathrm{mg} / \mathrm{kg}, \triangle$ for $5 \mathrm{mg} / \mathrm{kg}$, and + for $10 \mathrm{mg} / \mathrm{kg}$. 
beta1

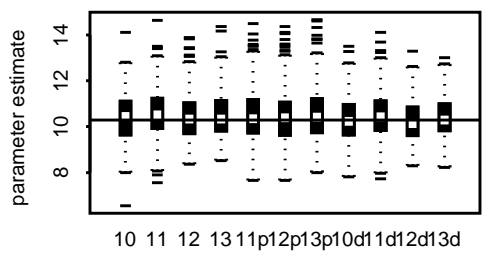

ec50g

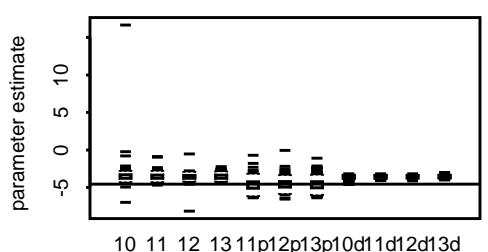

phi1

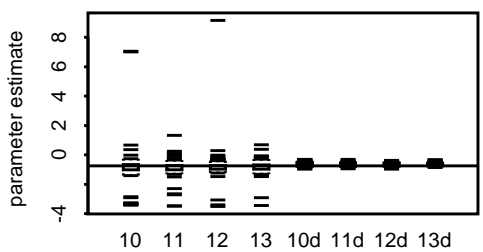

betag1

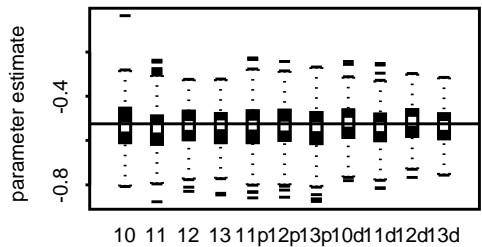

emax

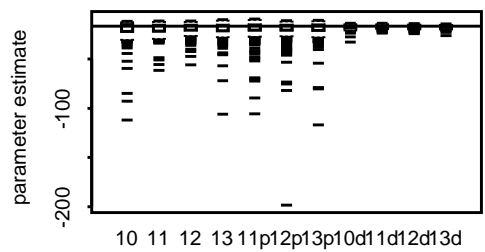

phi2

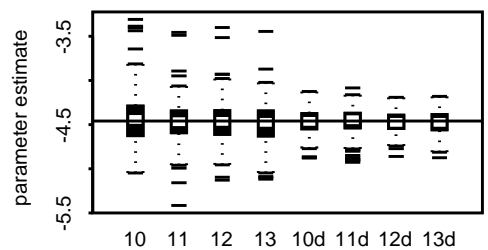

ec50

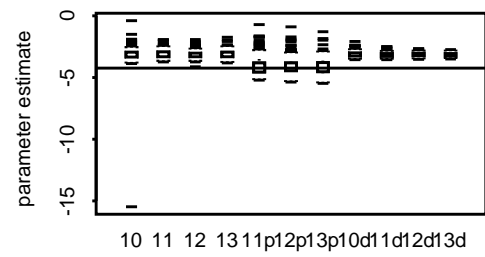

emaxg

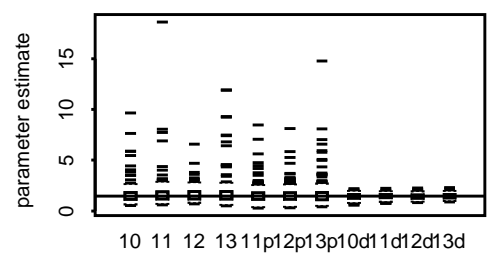

V

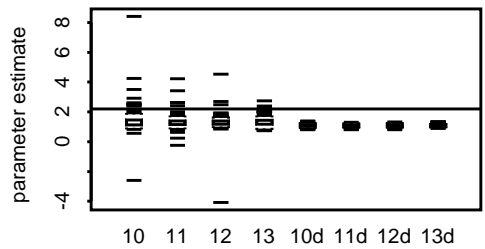

Figure 3: Box plots of the parameter estimates $\left(\beta, \beta_{\gamma}, E C_{50}, E C_{50_{\gamma}}, E_{\max }, E_{\max _{\gamma}}, \phi_{1}\right.$, and $\phi_{2}$ ) obtained from the study simulation in the case 10,11, 12, or 13 mice per dose level included in the study for either plasma concentration sampling ('p'), time-to-event sampling, or further dose exploration (' $d$ '). The horizontal line represents the parameter values used for the simulation. 